\title{
Biomechanical Analysis of the Dominant Foot and Non-Dominant Foot during Shooting with the Internal Instep as a Basis for Soccer Specific Exercises
}

\section{Introduction:}

\author{
Dr. Ibrahim Mohamed Farouk Gabr ${ }^{1}$ \\ Dr. Mansour Mohamed Ragheb Al-Maghraby ${ }^{2}$
}

Scientific research is a necessity for advances in all fields, including sport. Specific problems of sports performance are closely related to coaches' work. Therefore, coaches depend heavily on evaluating technical performance as a major tool in this respect.

Hammad, M. \& Kishk, H. (2004) indicated that distinguished and high technical performance in soccer according to the game description and technical requirements should be consistent with performance requirements and game characteristics. Shooting with internal instep in soccer requires coordination and harmony among agonist and antagonist muscles working on the arm, trunk and leg. (6: 32-33)

Mohamady, M. \& Ali, M. (1998) indicated various playing positions of soccer players and this leads to various attack and defense duties for each player according to his playing position. Therefore, soccer players should master all technical skills as most skills require that the player should use both feet (left and right) in coordination especially in shooting with internal instep (9: 255)

Alaa El-Din, G. \& Al-Sabbagh, N. (2007) indicated that biomechanical analysis of sports performance is a vital scientific method for improving performance and developing training programs (2: 7)

Hochmuth Gerard (1999) indicated that evaluating technical performance and using biomechanical analysis for skills help developing a vision about optimum performance (7: 78)

\section{Research Problem:}

Shooting with the internal instep is a basic soccer skill. It should be developed so as to be used effectively during the rare chances appearing for attackers during the match. Therefore, attackers, and soccer players in general, should concentrate on developing this skill for both feet (dominant and non-dominant).

After reviewing several matches of The Egyptian League during 2014-2015 season, the researchers noticed that Egyptian soccer players depend only on one foot, the dominant one, to perform all required skills, especially shooting with internal instep. Furthermore, they neglect the other, non-dominant- foot completely. This leads to losing the ball if the player is pivoting on his dominant foot during receiving the ball as is not able to shoot with his non-dominant foot. The player receives the ball and continues playing without using the non-dominant foot as he is discouraged to use it for fear of not achieving his playing objectives.

\footnotetext{
${ }^{1}$ Assistant Professor - Sports Training \& Kinesiology Department - Faculty of Physical Education Tanta University

${ }^{2}$ Lecturer - Team \& Racquet Sports Department - Faculty of Physical Education - Tanta University
} 
The researchers analyzed this observation through game analysis of some matches of the Egyptian League and Egypt's Cup. This analysis revealed that most Egyptian players are reluctant in using their non-dominant feet for performing major skills including shooting with internal instep. Most Egyptian players depend on their dominant feet while players of European Leagues perform shooting with internal instep with both feet.

Game situations require that soccer players should use both feet effectively as in some situations the player may initiate attack from various places in the field. Thus he needs to use his non-dominant foot. If the player does not use his non-dominant hand he may lose the right moment for shooting in addition to losing the ball. Modern soccer needs speed and fast reaction to deal with the ball accurately and without this, players may not be able to score goals to win.

This indicates the importance of using non-dominant foot in shooting with internal instep as a means for scoring goals, the final target of any attack in soccer.

The researchers thought in using biomechanical analysis to identify the weaknesses of the correct technique for using non-dominant foot in shooting with internal instep. This can be done through analyzing the dominant (kicking) foot and non-dominant (non-kicking) foot for right-footed and left-footed players.

This is consistent with previous studies of Souilem, A. (2013), Saber, T. (2011) and Abd El-Aziz, A. (2009) (4, 11 and 1)

Biomechanics specialists like Berekaa, M. \& Al-Sokary, K. (2002) indicated that performance analysis identifies weaknesses and strengths of the technique used by an athlete and this helps coaches to design the type of training suitable for their athletes. Alaa El-Din, G. (1989) indicated that mastering and improving technical performance is closely related to the correct details of movement and these fine details are unified in one locomotion system used in technical performance in addition to how individual athletes direct and control these movements. Movements of all body parts are unified in one system directing movements so that all performances or motor behaviors are integrated $(5: 29)(3: 3,5)$

According to the researchers' knowledge, no previous studies dealt with biomechanical indicators distinguishing dominant and non-dominant foot during shooting with internal instep in soccer. In addition, coaches do not use these indicators in designing their training programs. This led the researchers to perform the current research to study the biomechanical indicators of dominant and non-dominant foot during shooting with internal instep in soccer as a basis for designing specific exercises training program for soccer players.

Aim:

The current research aims to establish a biomechanical basis for specific exercises of shooting with internal instep in soccer through identifying the following:

1. The biomechanical characteristics of the dominant and non-dominant foot during shooting with internal instep for right-footed soccer players

2. The biomechanical characteristics of the dominant and non-dominant foot during shooting with internal instep for left-footed soccer players 


\section{Hypotheses:}

1. There are statistically significant differences between the dominant and nondominant foot during shooting with internal instep for right-footed soccer players

2. There are statistically significant differences between the dominant and nondominant foot during shooting with internal instep for left-footed soccer players

\section{Methods:}

\section{$\underline{\text { Research Design }}$}

The researchers used descriptive approach through biomechanical analysis, Preliminary investigation Preliminary investigation was performed on 5-1-2015 (12 P.m.) at the main court of Faculty of Physical Education - Tanta University on a pilot sample (one player) from the same research community and outside the main sample to identify the steps of motion analysis of the skill under investigation, and main study was performed on 19-1-2015 (5:00 P.m.) at the main court of Faculty of Physical Education - Tanta University. Procedures of analysis were as follows:

\section{Participants:}

Research community included all junior soccer players less than (19) years) who play in soccer zones of the Egyptian Football Federation.

Research sample was purposefully chosen from the first team of ALMOKAWELOUN ALARAB sports club including (3) attackers (two left-footed and one right-footed) and the first team of PETROJET sports club including (3) attackers (two right-footed and one left-footed). Sample was chosen according to the following criteria:

- All players are registered in the Egyptian Football Federation

- All players are distinguished in performing shooting with the internal instep

- All players are free of injuries

\section{Data Collection Tools:}

- Dmas 7 motion analysis device

- One SONY camera (120f/sec) with tripod

- Calibration device including (6) points for 2D analysis

- Phosphoric markers and white (medical) bandages to mark joint points

- A computer set and cables

Preliminary investigation:

Preliminary investigation was performed on 5-1-2015 (12 P.m.) at the main court of Faculty of Physical Education - Tanta University on a pilot sample (one player) from the same research community and outside the main sample to identify the steps of motion analysis of the skill under investigation.

Preliminary investigation aimed to verify the suitability of place and time of recording and places of sticking markers to body joints. In addition, the Preliminary investigation aimed to identify the place, altitude and distance of the camera. Furthermore, the Preliminary investigation aimed to identify the steps to be performed by players, number of trials, rest intervals and place of scale. 
Results indicated the suitability of the place but timing was changed to $(5: 00$ $\mathrm{pm})$ to avoid shadows. All markers were clear. Camera distance was set on $(7.90 \mathrm{~m})$ and focus altitude from ground was $(1.10 \mathrm{~m})$. Place of scale was identified.

Main Study:

Main study was performed on 19-1-2015 (5:00 P.m.) at the main court of Faculty of Physical Education - Tanta University. Procedures of analysis were as follows:

1. All players were prepared inside the main court of Faculty of Physical Education - Tanta University through warm-up and marking joint points.

2. Cameras $(120 \mathrm{f} / \mathrm{sec})$ were used and the scale was set inside the optical range of camera. The camera was set on the right side of players (distance $=7.90 \mathrm{~m}-$ focus altitude $=1.10 \mathrm{~m}$ )

3. Three trials for each player were recorded and the best attempt was used for analysis

4. Motion analysis software was used to analyze the trials

Statistical Treatment:

The researchers used SPSS software to calculate the following: Mean $-\mathrm{SD}-(\mathrm{t})$ test to Clarification The Significance of Differences between the dominant and nondominant foot on all research variables for the right-footed player, and the dominant and non-dominant foot on all research variables for the left-footed player.

\section{Results:}

Table (1): The biomechanical characteristics of Averages knee, thigh and foot angles for right-footed (dominant) player

\begin{tabular}{|c|c|c|c|c|c|c|c|c|}
\hline \multicolumn{2}{|c|}{ Variables } & \multirow{2}{*}{$\begin{array}{c}\begin{array}{c}\text { Measur } \\
\text { ement }\end{array} \\
\text { DEG }\end{array}$} & \multirow{2}{*}{$\begin{array}{c}\begin{array}{r}\text { Knee } \\
\text { angle } \\
(\mathbf{L})\end{array} \\
136.7\end{array}$} & \multirow{2}{*}{$\begin{array}{c}\begin{array}{c}\text { Knee } \\
\text { angle (R) }\end{array} \\
128.8\end{array}$} & \multirow{2}{*}{$\begin{array}{c}\begin{array}{c}\text { Thigh } \\
\text { angle }(\mathbf{L})\end{array} \\
163.9\end{array}$} & \multirow{2}{*}{$\begin{array}{c}\begin{array}{c}\text { Thigh } \\
\text { angle }(\mathbf{R})\end{array} \\
149.8\end{array}$} & \multirow{2}{*}{$\begin{array}{c}\begin{array}{c}\text { Foot } \\
\text { angle } \\
\text { (L) }\end{array} \\
106.8\end{array}$} & \multirow{2}{*}{$\begin{array}{r}\begin{array}{r}\text { Foot } \\
\text { angle } \\
(\mathbf{R})\end{array} \\
98.34\end{array}$} \\
\hline Prelimina & $\begin{array}{l}\text { Right foot } \\
\text { (dominant) }\end{array}$ & & & & & & & \\
\hline $\begin{array}{c}\text { ry stage } \\
\text { (swing) }\end{array}$ & $\begin{array}{c}\text { Left foot } \\
\text { (non- } \\
\text { dominant) } \\
\end{array}$ & DEG & 78.59 & 161.1 & 156.6 & 171.6 & 96.06 & 90.0 \\
\hline \multirow{2}{*}{$\begin{array}{c}\text { Basic } \\
\text { stage } \\
\text { (contact) }\end{array}$} & $\begin{array}{l}\text { Right foot } \\
\text { (dominant) }\end{array}$ & DEG & 140.5 & 185.2 & 139.5 & 140.8 & 85.98 & 119.7 \\
\hline & $\begin{array}{c}\text { Left foot } \\
\text { (non- } \\
\text { dominant) }\end{array}$ & DEG & 186.3 & 146.9 & 136.8 & 189.7 & 83.42 & 84.81 \\
\hline \multirow{2}{*}{$\begin{array}{c}\text { Final } \\
\text { stage } \\
\text { (follow- } \\
\text { up) }\end{array}$} & $\begin{array}{l}\text { Right foot } \\
\text { (dominant) }\end{array}$ & DEG & 137.0 & 162.3 & 136.8 & 124.3 & 93.42 & 118.2 \\
\hline & $\begin{array}{c}\text { Left foot } \\
\text { (non- } \\
\text { dominant) }\end{array}$ & DEG & 111.1 & 122.4 & 76.29 & 123.6 & 70.16 & 82.76 \\
\hline
\end{tabular}




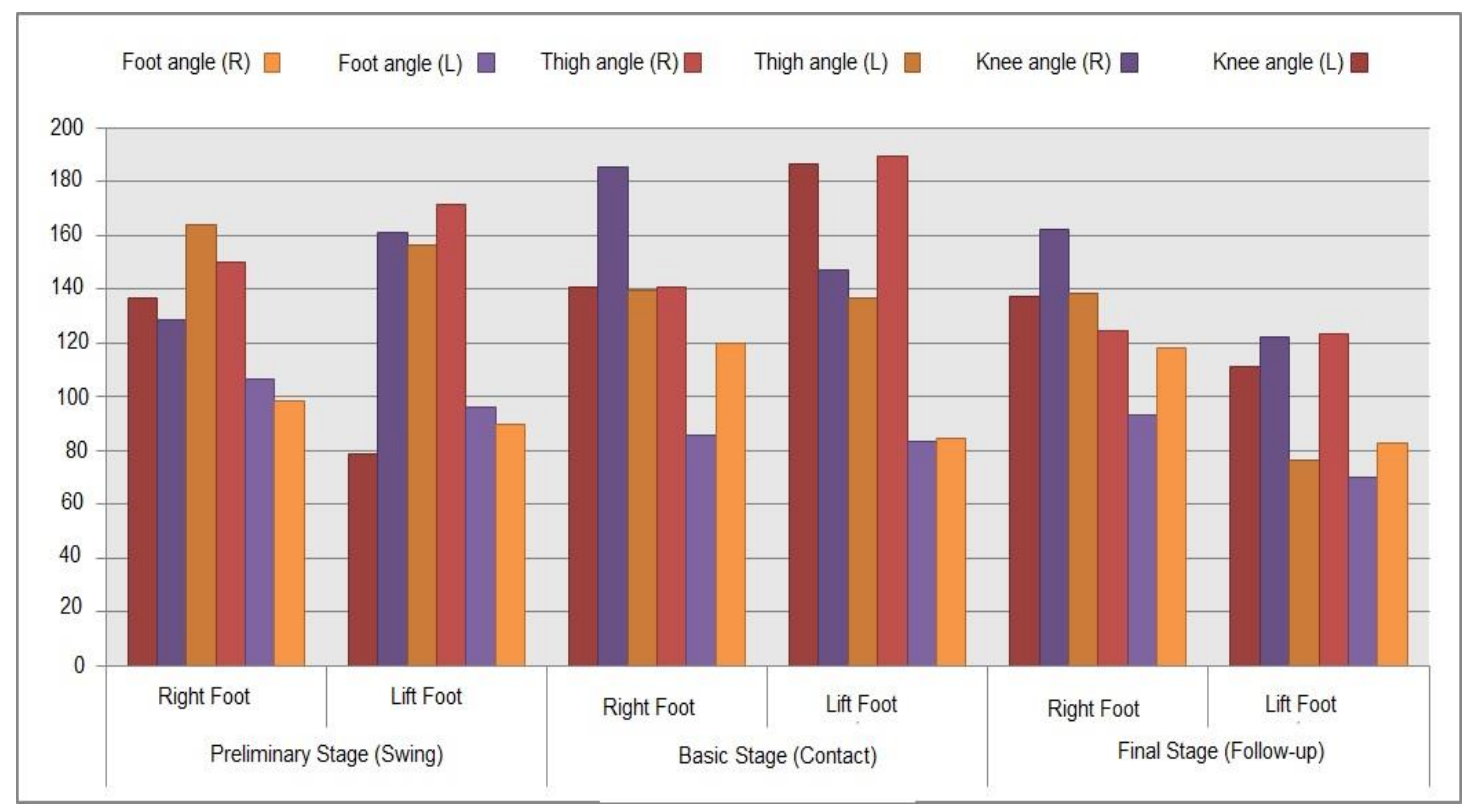

Figure (1): The biomechanical characteristics of Averages knee, thigh and foot angles for right-footed (dominant) player

Table (2): The biomechanical characteristics of Averages The weight of the body center for right-footed (dominant) player

\begin{tabular}{c|c|c|c|c|c|c|c}
\hline \hline & Variables & $\begin{array}{c}\text { CG } \\
\text { Horizontal } \\
\text { Velocity }\end{array}$ & $\begin{array}{c}\text { CG } \\
\text { Vertical } \\
\text { Velocity }\end{array}$ & $\begin{array}{c}\text { CG } \\
\text { Horizontal } \\
\text { Acceleratio } \\
\mathbf{n}\end{array}$ & $\begin{array}{c}\text { CG } \\
\text { Vertical } \\
\text { Accelera } \\
\text { tion }\end{array}$ & $\begin{array}{c}\text { CG } \\
\text { Resultant } \\
\text { Momentu } \\
\text { m }\end{array}$ & $\begin{array}{c}\text { CG } \\
\text { Resultant } \\
\text { Force }\end{array}$ \\
\hline \hline \multicolumn{2}{|c|}{ Measurements } & {$[\mathrm{m} / \mathrm{s}]$} & {$[\mathrm{m} / \mathrm{s}]$} & {$\left[\mathrm{m} / \mathrm{s}^{\wedge} 2\right]$} & {$\left[\mathrm{m} / \mathrm{s}^{\wedge} 2\right]$} & {$[\mathrm{kg} \mathrm{m} / \mathrm{s}]$} & {$[\mathrm{N}]$} \\
\hline \hline \multirow{2}{*}{$\begin{array}{c}\text { Prelimin } \\
\text { ary stage } \\
\text { (swing) }\end{array}$} & Right foot (dominant) & 4.79 & 0.4145 & 13.580 & 13.25 & 355.80 & 1404.0 \\
\cline { 2 - 8 } & $\begin{array}{c}\text { Left foot (non- } \\
\text { dominant) }\end{array}$ & -4.513 & -0.4646 & 7.493 & 8.631 & 385.4 & 901.9 \\
\hline \hline $\begin{array}{c}\text { Basic } \\
\text { stage } \\
\text { (contact) }\end{array}$ & Right foot (dominant) & 4.122 & 1.598 & 9.842 & 12.91 & 327.1 & 1201.0 \\
\cline { 2 - 9 } & $\begin{array}{c}\text { Left foot (non- } \\
\text { dominant) }\end{array}$ & -2.382 & 0.877 & 5.145 & 9.096 & 219.6 & 825.5 \\
\hline $\begin{array}{c}\text { Final } \\
\text { stage } \\
\text { (follow- } \\
\text { up) }\end{array}$ & Right foot (dominant) & 4.081 & 1.350 & 5.278 & 13.80 & 302.6 & 1093.0 \\
\cline { 2 - 9 } & Left foot (non- & -2.942 & -0.1791 & -1.713 & 11.98 & 236.2 & 946.6 \\
\hline \hline
\end{tabular}




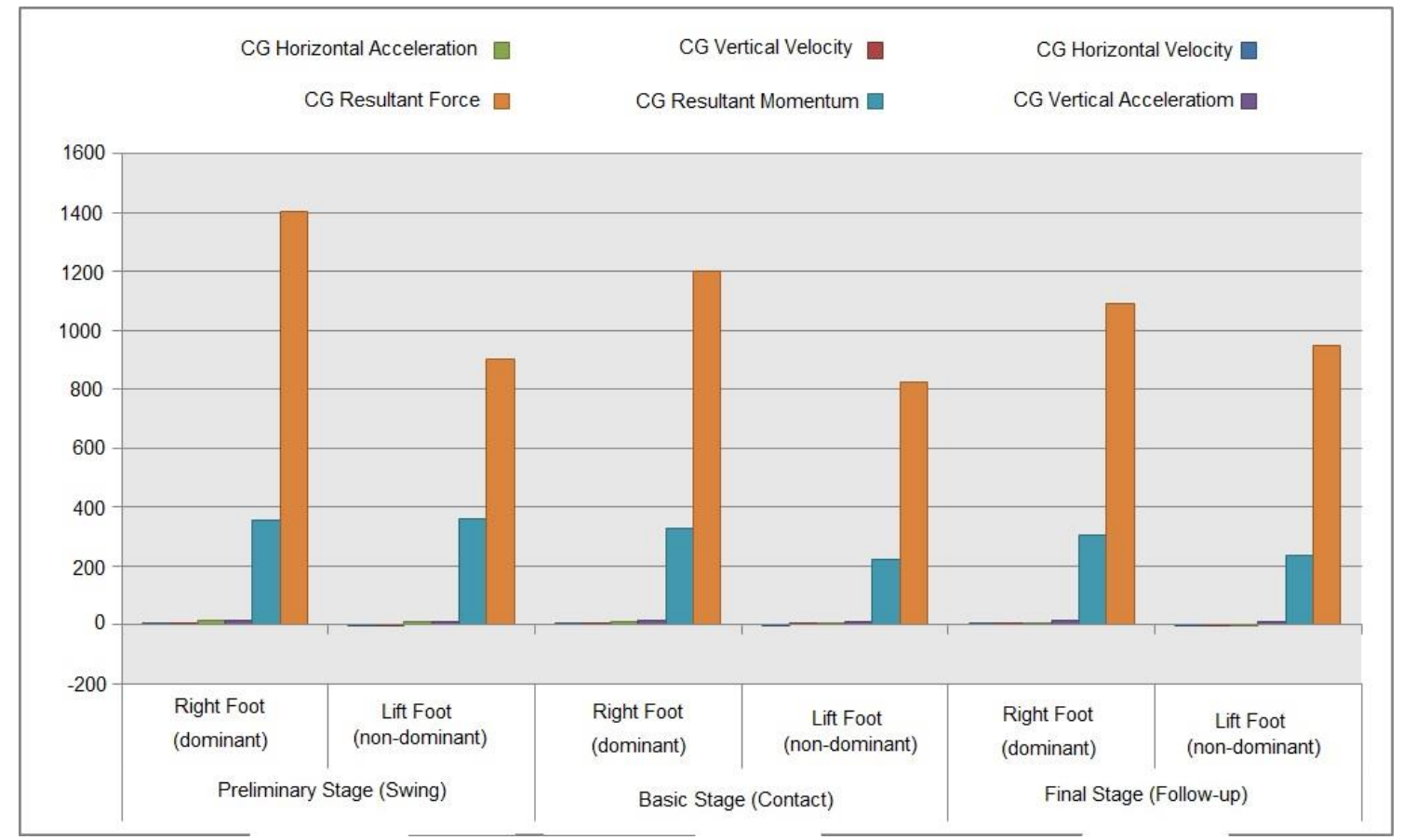

Figure (2): The biomechanical characteristics of Averages The weight of the body center for right-footed (dominant) player

Table (3): The biomechanical characteristics of Averages knee, thigh and foot angles for left-footed (dominant) player

\begin{tabular}{|c|c|c|c|c|c|c|c|c|}
\hline \multicolumn{2}{|c|}{ Variables } & $\begin{array}{c}\text { Measu } \\
\text { remen } \\
\mathbf{t} \\
\end{array}$ & $\begin{array}{c}\text { Knee } \\
\text { angle } \\
\text { (L) } \\
\end{array}$ & $\begin{array}{c}\text { Knee } \\
\text { angle }(\mathbf{R})\end{array}$ & $\begin{array}{c}\text { Thigh } \\
\text { angle (L) }\end{array}$ & $\begin{array}{c}\text { Thigh } \\
\text { angle (R) }\end{array}$ & $\begin{array}{c}\text { Foot } \\
\text { angle } \\
\text { (L) } \\
\end{array}$ & $\begin{array}{c}\text { Foot } \\
\text { angle } \\
(\mathbf{R}) \\
\end{array}$ \\
\hline \multirow{2}{*}{$\begin{array}{c}\text { Preliminary } \\
\text { stage } \\
\text { (swing) }\end{array}$} & $\begin{array}{c}\text { Left foot } \\
\text { (dominant) }\end{array}$ & DEG & 160.6 & 159.2 & 199.2 & 146.2 & 116.8 & 102.6 \\
\hline & $\begin{array}{l}\text { Right foot } \\
\text { (non- } \\
\text { dominant) }\end{array}$ & DEG & 150.9 & 66.6 & 157.1 & 136.4 & 99.09 & 83.89 \\
\hline \multirow{2}{*}{$\begin{array}{c}\text { Basic stage } \\
\text { (contact) }\end{array}$} & $\begin{array}{c}\text { Left foot } \\
\text { (dominant) }\end{array}$ & DEG & 168.5 & 146.8 & 164.8 & 132.6 & 96.09 & 74.4 \\
\hline & $\begin{array}{c}\text { Right foot } \\
\text { (non- } \\
\text { dominant) }\end{array}$ & DEG & 124.4 & 133.2 & 144.4 & 118.1 & 88.54 & 62.94 \\
\hline \multirow{2}{*}{$\begin{array}{l}\text { Final stage } \\
\text { (follow-up) }\end{array}$} & $\begin{array}{c}\text { Left foot } \\
\text { (dominant) }\end{array}$ & DEG & 135.0 & 127.7 & 141.7 & 137.4 & 103.7 & 74.87 \\
\hline & $\begin{array}{l}\text { Right foot } \\
\text { (non- } \\
\text { dominant) }\end{array}$ & DEG & 142.9 & 118.9 & 142.6 & 65.84 & 88.16 & 63.82 \\
\hline
\end{tabular}




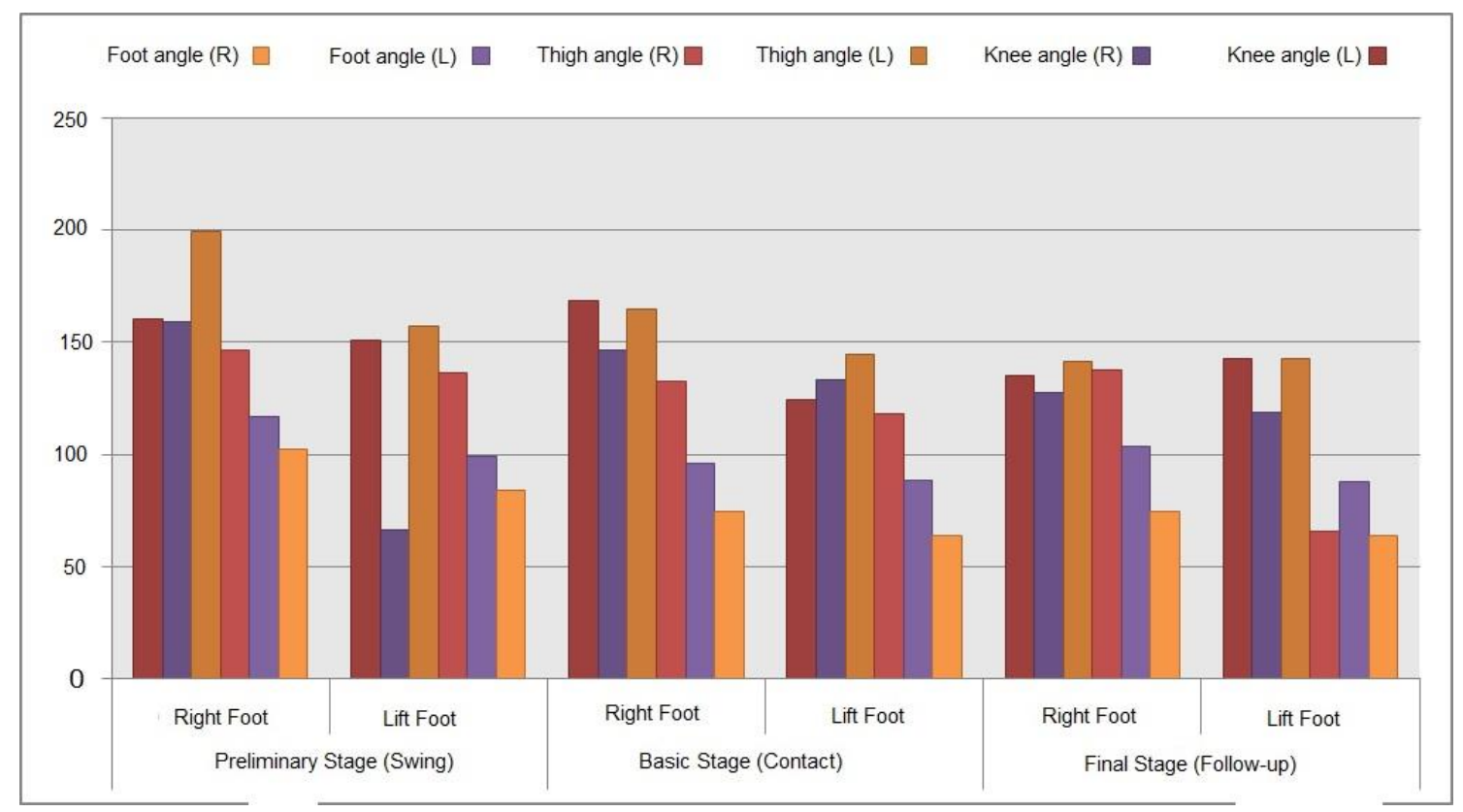

Figure (3): The biomechanical characteristics of Averages knee, thigh and foot angles for left-footed (dominant) player

Table (4): The biomechanical characteristics of Averages The weight of the body center for left-footed (dominant) player

\begin{tabular}{|c|c|c|c|c|c|c|c|}
\hline & Variables & $\begin{array}{l}\text { CG } \\
\text { Horizonta } \\
\text { I Velocity }\end{array}$ & $\begin{array}{c}\text { CG } \\
\text { Vertical } \\
\text { Velocity }\end{array}$ & $\begin{array}{c}\text { CG } \\
\text { Horizonta } \\
1 \\
\text { Accelerati } \\
\text { on } \\
\end{array}$ & $\begin{array}{c}\text { CG } \\
\text { Vertical } \\
\text { Accelerati } \\
\text { on }\end{array}$ & $\begin{array}{c}\text { CG } \\
\text { Resultant } \\
\text { Momentu } \\
\text { m }\end{array}$ & $\begin{array}{c}\text { CG } \\
\text { Resultant } \\
\text { Force }\end{array}$ \\
\hline \multicolumn{2}{|c|}{$\begin{array}{l}\text { Measurements } \\
\end{array}$} & {$[\mathrm{m} / \mathrm{s}]$} & {$[\mathrm{m} / \mathrm{s}]$} & {$\left[\mathrm{m} / \mathrm{s}^{\wedge} 2\right]$} & {$\left[\mathrm{m} / \mathrm{s}^{\wedge} 2\right]$} & {$[\mathrm{kg} \mathrm{m} / \mathrm{s}]$} & {$[\mathrm{N}]$} \\
\hline \multirow{2}{*}{$\begin{array}{l}\text { Prelimina } \\
\text { ry stage } \\
\text { (swing) }\end{array}$} & Left foot (dominant) & 6.646 & 1.253 & 0.3875 & 7.347 & 500.5 & 1808.0 \\
\hline & $\begin{array}{c}\begin{array}{l}\text { Right foot (non- } \\
\text { dominant) }\end{array} \\
\end{array}$ & 4.868 & -0.6363 & 21.91- & -3.839 & 360.8 & 97.00 \\
\hline \multirow{2}{*}{$\begin{array}{c}\text { Basic } \\
\text { stage } \\
\text { (contact) }\end{array}$} & Left foot (dominant) & 6.685 & 0.9765 & 12.23 & 9.635 & 357.9 & 1378.0 \\
\hline & $\begin{array}{c}\text { Right foot (non- } \\
\text { dominant) }\end{array}$ & 2.93 & -0.892 & $13.35-$ & -12.53 & 226.7 & 574.2 \\
\hline \multirow{2}{*}{$\begin{array}{c}\text { Final } \\
\text { stage } \\
\text { (follow- } \\
\text { up) }\end{array}$} & Left foot (dominant) & 5.488 & 0.9604 & -1.123 & -6.817 & 412.3 & 1065.0 \\
\hline & $\begin{array}{l}\text { Right foot (non- } \\
\text { dominant) }\end{array}$ & 2.438 & -0.2238 & -1.14 & -12.47 & 220.3 & 936.4 \\
\hline
\end{tabular}




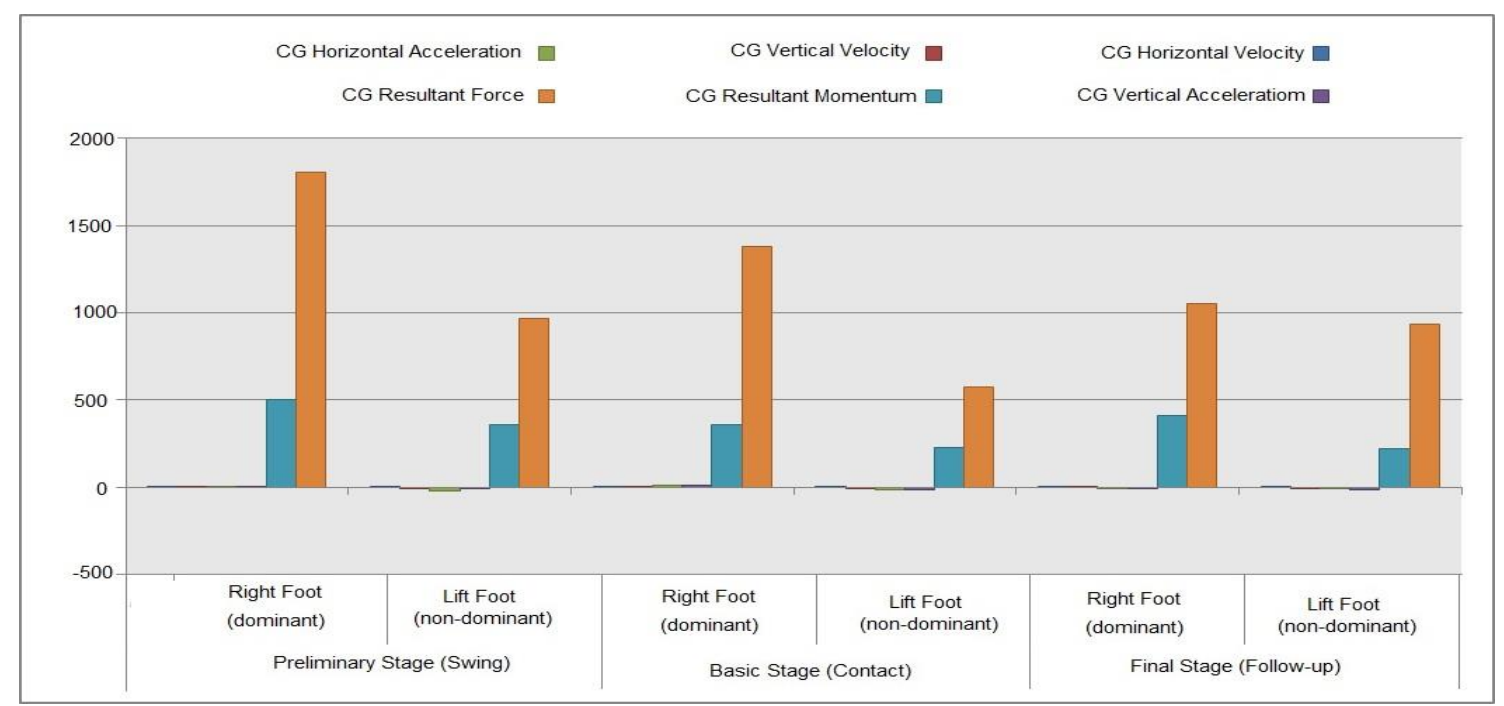

Figure (4): The biomechanical characteristics of Averages The weight of the body center for left-footed (dominant) player

Table (5): The Significance of Differences between the dominant and nondominant foot on all research variables for the right-footed player

\begin{tabular}{|c|c|c|c|c|c|c|}
\hline \multirow{2}{*}{ Variables } & \multicolumn{2}{|c|}{ Dominant foot (right) } & \multicolumn{2}{|c|}{ Non-dominant foot (left) } & \multirow{2}{*}{$\begin{array}{c}\text { Means } \\
\text { difference }\end{array}$} & \multirow{2}{*}{$(\mathbf{t})$} \\
\hline & Mean & SD \pm & Mean & SD \pm & & \\
\hline Knee angle (L) & 138.06 & 2.112 & 125.33 & 4.898 & 12.73 & $* 4.135$ \\
\hline Knee angle $(\mathrm{R})$ & 158.76 & 28.365 & 143.46 & 19.577 & 15.30 & *3.827 \\
\hline Thigh angle $(\mathrm{L})$ & 147.33 & 14.366 & 123.23 & 41.839 & 24.10 & $* 5.568$ \\
\hline Thigh angle ( $\mathrm{R})$ & 138.30 & 12.932 & 161.63 & 34.158 & -23.33 & $* 5.889$ \\
\hline Foot angle $(\mathrm{L})$ & 95.40 & 10.550 & 83.210 & 12.950 & 12.19 & $* 4.356$ \\
\hline Foot angle $(\mathrm{R})$ & 112.08 & 11.992 & 85.85 & 3.730 & 26.23 & $* 11.484$ \\
\hline CG Horizontal Velocity & 4.331 & 0.4074 & -3.279 & 1.098 & 7.610 & $* 10.748$ \\
\hline CG Vertical Velocity & 1.120 & 0.622 & -0.506 & 0.349 & 1.626 & $* 2.862$ \\
\hline CG Horizontal Acceleration & 9.560 & 4.156 & 3.641 & 4.783 & 5.919 & $* 3.340$ \\
\hline CG Vertical Acceleration & 13.32 & 0.447 & 9.896 & 1.820 & 3.424 & $* 3.943$ \\
\hline CG Resultant Momentum & 328.5 & 26.632 & 271.4 & 75.80 & 57.10 & $* 10.749$ \\
\hline CG Resultant Force & 1232.66 & 157.899 & 891.33 & 20.547 & 341.33 & $* 44.257$ \\
\hline
\end{tabular}

(t) Table value on $P \leq 0.05=2.776$

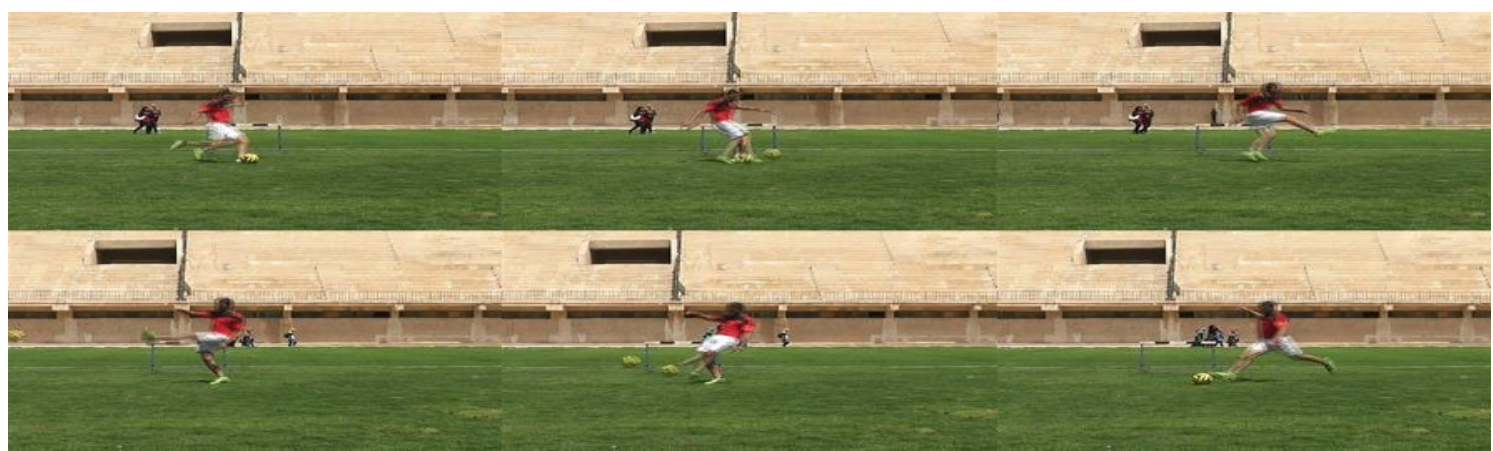

Figure (5): The Significance of Differences between the dominant and nondominant foot on all research variables for the right-footed player 


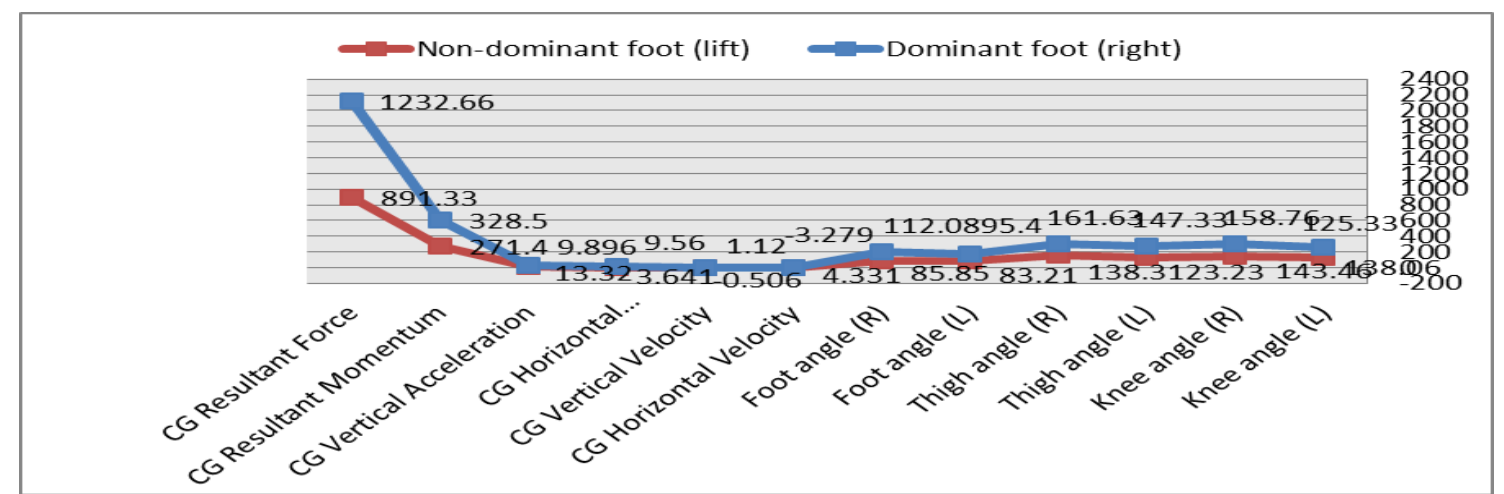

Figure (6): The Significance of Differences between the dominant and nondominant foot on all research variables for the right-footed player

Table (5) indicates statistically significant differences between the dominant and non-dominant foot for all participants as $(\mathrm{t})$ calculated value ranged between (2.862) and (44.257) which exceeds its table value.

Table (6): The Significance of Differences between the dominant and nondominant foot on all research variables for the left-footed player

\begin{tabular}{|c|c|c|c|c|c|c|}
\hline \multirow{2}{*}{ Variables } & \multicolumn{2}{|c|}{ Dominant foot (right) } & \multicolumn{2}{|c|}{ Non-dominant foot (left) } & \multirow{2}{*}{$\begin{array}{c}\text { Means } \\
\text { difference }\end{array}$} & \multirow{2}{*}{ (t) } \\
\hline & Mean & $\mathrm{SD} \pm$ & Mean & $\mathrm{SD} \pm$ & & \\
\hline Knee angle $(\mathrm{L})$ & 154.7 & 17.511 & 139.4 & 13.592 & 15.30 & $* 4.753$ \\
\hline Knee angle (R) & 144.56 & 15.86 & 106.23 & 35.06 & 38.33 & $* 9.305$ \\
\hline Thigh angle $(\mathrm{L})$ & 168.56 & 28.934 & 148.03 & 7.903 & 20.53 & $* 5.860$ \\
\hline Thigh angle $(\mathrm{R})$ & 138.73 & 6.897 & 106.78 & 36.616 & 31.95 & $* 8.390$ \\
\hline Foot angle (L) & 105.53 & 10.475 & 91.93 & 6.202 & 13.60 & $* 5.713$ \\
\hline Foot angle $(\mathbf{R})$ & 83.95 & 16.147 & 70.55 & 11.55 & 13.40 & $* 4.410$ \\
\hline CG Horizontal Velocity & 6.273 & 0.679 & 3.412 & 1.284 & 2.861 & $* 3.540$ \\
\hline CG Vertical Velocity & 1.063 & 0.161 & -0.584 & 0.334 & 1.647 & $* 4.076$ \\
\hline CG Horizontal Acceleration & 3.831 & 7.312 & -12.13 & 10.437 & 15.961 & $* 6.562$ \\
\hline CG Vertical Acceleration & 3.388 & 8.911 & -9.613 & 5.002 & 13.001 & *6.038 \\
\hline CG Resultant Momentum & 423.56 & 71.96 & 269.26 & 79.33 & 154.30 & $* 21.732$ \\
\hline CG Resultant Force & 1414.0 & 11.930 & 826.86 & 219.45 & 587.14 & $* 66.857$ \\
\hline
\end{tabular}

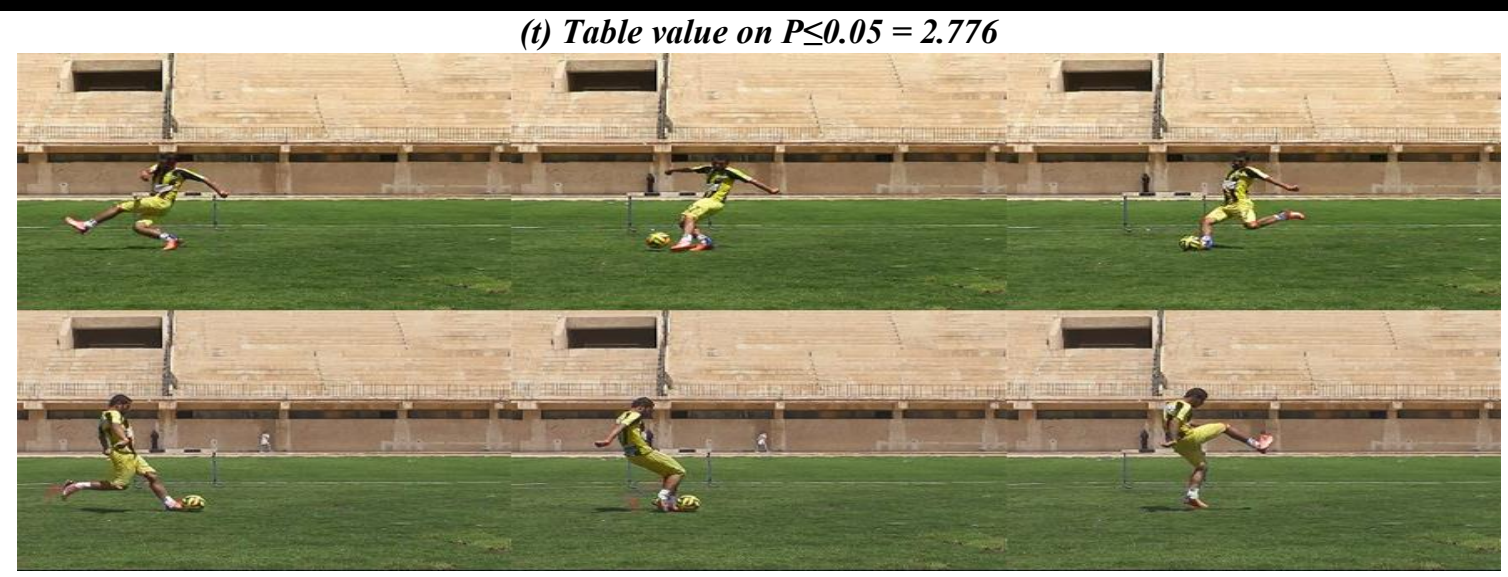

Figure (7): The Significance of Differences between the dominant and nondominant foot on all research variables for the left-footed player 


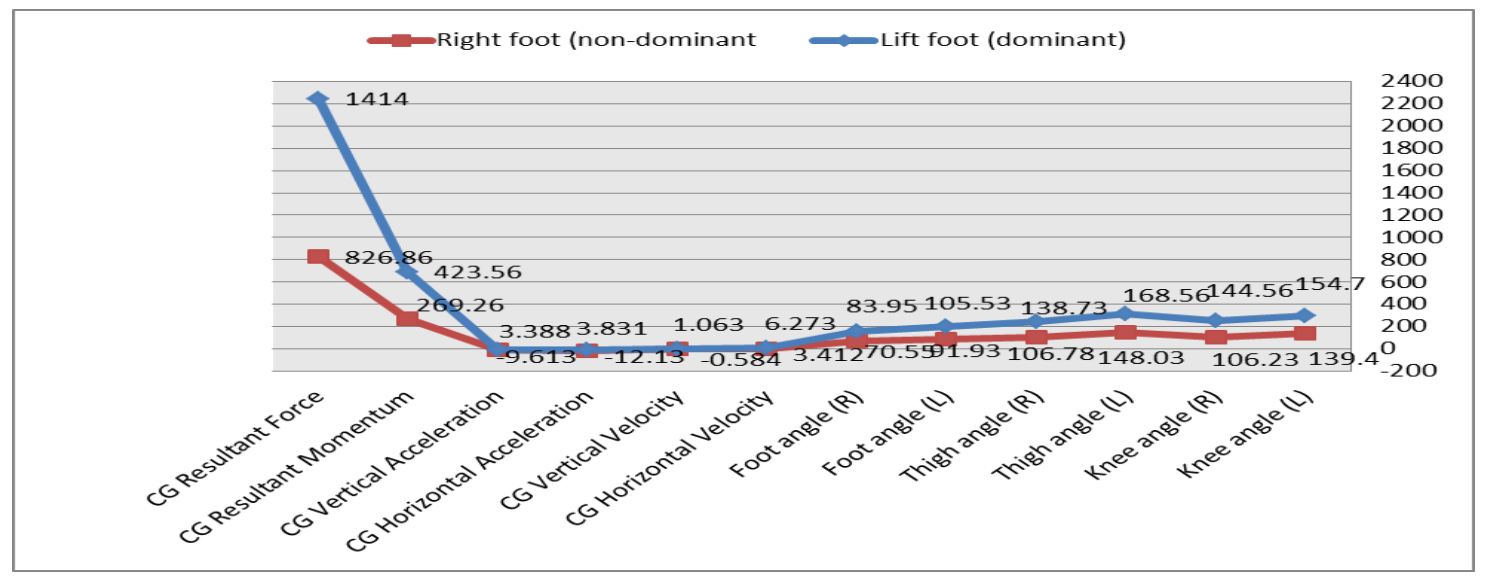

Figure (8): The Significance of Differences between the dominant and nondominant foot on all research variables for the left-footed player

Table (6) indicates statistically significant differences between the dominant and non-dominant foot for all participants as $(\mathrm{t})$ calculated value ranged between (3.540) and (66.857) which exceeds its table value on $\mathrm{P} \leq 0.05$.

Results shown in table (5) indicate statistically significant differences between the dominant (right) and non-dominant (left) foot as follows:

- Knee angle (L) as (t) calculated values (4.135) exceeds its table value (2.776) on $\mathrm{P} \leq 0.05$

- Knee angle $(\mathrm{R})$ as (t) calculated values (3.827) exceeds its table value (2.776) on $\mathrm{P} \leq 0.05$

- Thigh angle (L) as (t) calculated values (5.568) exceeds its table value (2.776) on $\mathrm{P} \leq 0.05$

- $\quad$ Thigh angle $(\mathrm{R})$ as $(\mathrm{t})$ calculated values (5.889) exceeds its table value (2.776) on $\mathrm{P} \leq 0.05$

- Foot angle (L) as (t) calculated values (4.356) exceeds its table value (2.776) on $\mathrm{P} \leq 0.05$

- Foot angle (R) as (t) calculated values (11.484) exceeds its table value (2.776) on $\mathrm{P} \leq 0.05$

- CG horizontal velocity as (t) calculated values (10.748) exceeds its table value (2.776) on $\mathrm{P} \leq 0.05$

- CG vertical velocity as (t) calculated values (2.862) exceeds its table value (2.776) on $\mathrm{P} \leq 0.05$

- CG horizontal acceleration as (t) calculated values (3.430) exceeds its table value (2.776) on $\mathrm{P} \leq 0.05$

- CG vertical acceleration as (t) calculated values (3.943) exceeds its table value (2.776) on $\mathrm{P} \leq 0.05$

- CG resultant momentum as (t) calculated values (10.749) exceeds its table value (2.776) on $\mathrm{P} \leq 0.05$

- CG resultant force as (t) calculated values (44.257) exceeds its table value (2.776) on $\mathrm{P} \leq 0.05$ 
Table (6) indicates statistically significant differences between the dominant and non-dominant foot for the left-footed players. These values were derived from biomechanical analysis of left-footed players' performances of shooting with internal instep with dominant (left) and non-dominant (right) foot as seen in tables ( $3 \& 4)$ and figures $(3 \& 4)$.

Results shown in table (6) indicate statistically significant differences between the dominant (left) and non-dominant (right) foot as follows:

- Knee angle (L) as (t) calculated values (4.735) exceeds its table value (2.776) on $\mathrm{P} \leq 0.05$

- Knee angle $(\mathrm{R})$ as $(\mathrm{t})$ calculated values (9.305) exceeds its table value (2.776) on $\mathrm{P} \leq 0.05$

- Thigh angle $(\mathrm{L})$ as $(\mathrm{t})$ calculated values (5.860) exceeds its table value (2.776) on $\mathrm{P} \leq 0.05$

- Thigh angle $(\mathrm{R})$ as $(\mathrm{t})$ calculated values (8.390) exceeds its table value (2.776) on $\mathrm{P} \leq 0.05$

- Foot angle (L) as (t) calculated values (5.713) exceeds its table value (2.776) on $\mathrm{P} \leq 0.05$

- Foot angle (R) as (t) calculated values (4.410) exceeds its table value (2.776) on $\mathrm{P} \leq 0.05$

- CG horizontal velocity as (t) calculated values (3.540) exceeds its table value (2.776) on $\mathrm{P} \leq 0.05$

- CG vertical velocity as (t) calculated values (4.076) exceeds its table value (2.776) on $\mathrm{P} \leq 0.05$

- CG horizontal acceleration as (t) calculated values (6.562) exceeds its table value (2.776) on $\mathrm{P} \leq 0.05$

- CG vertical acceleration as (t) calculated values (3.038) exceeds its table value (2.776) on $\mathrm{P} \leq 0.05$

- CG resultant momentum as (t) calculated values (21.732) exceeds its table value (2.776) on $\mathrm{P} \leq 0.05$

- CG resultant force as (t) calculated values (66.857) exceeds its table value (2.776) on $\mathrm{P} \leq 0.05$

\section{Discussion:}

Table (5) indicates statistically significant at value on $\mathrm{P} \leq 0.05$ differences between the dominant and non-dominant foot for the right-footed players on the following variables: knee angle $(\mathrm{L})$ - knee angle $(\mathrm{R})$ - thigh angle $(\mathrm{L})$ - thigh angle $(\mathrm{R})$ - foot angle (L) - foot angle (R) - CG horizontal velocity - CG vertical velocity - CG horizontal acceleration - $\mathrm{CG}$ vertical acceleration - $\mathrm{CG}$ resultant momentum - CG resultant force). These values were derived from biomechanical analysis of right-footed players' performances of shooting with internal instep with dominant (right) and nondominant (left) foot as seen in tables (1\&2) and figures (1\&2).

The researchers think that these differences are due to the lack of balance in size and energy generation at the lower body muscles. In addition, there is a clear lack of coordination between the dominant and non-dominant foot. Mohamady, M. \& Ali, M. (1998) indicated that when players attempt to shoot with the dominant foot only, they 
continue doing so without thinking of using the non-dominant foot for fear of failing in scoring a goal (11)

Hammad, M. \& Kishk, H. (2004) indicated that shooting with internal instep in soccer requires coordination and harmony among agonist and antagonist muscles working on the arm, trunk and leg. (6)

Tables (1) and (3) indicated that right-footed players do not improve their left knee angle, left thigh angle or left foot angle and vice versa for the left-footed players. Biomechanical analysis of both types of players revealed great variance in these angles between the dominant and non-dominant foot.

Tables (2) and (4) indicated that soccer coaches are not interested in specific exercises for the non-dominant foot during the training unit. Biomechanical analysis of both types of players indicated greater variance in vertical velocity, horizontal velocity, vertical acceleration, horizontal acceleration, resultant momentum and resultant force between the dominant and non-dominant foot in both types of players.

Hussam El-Din, T. (1993) indicated that full knowledge of information related to human movement through anatomy, physiology, biology and biomechanics is vital for improving motor performance (8: 47).

\section{Conclusions:}

In the light of this research aim, hypotheses, methodology and results, the researchers concluded the following:

- Results also indicate statistically significant differences between the dominant (right) and non-dominant (left) foot in Favour the dominant (right) foot of as follows: (from high to low) was as follows: CG resultant force - Foot angle (L) CG resultant momentum - CG horizontal velocity - Thigh angle (R) - Thigh angle (L) - Foot angle (R) - Knee angle (L) - CG vertical acceleration - Knee angle (R) - CG horizontal acceleration - CG vertical velocity. These results prove the first hypothesis.

- Results also indicated statistically significant differences between the dominant (left) and non-dominant (right) foot in Favour the dominant (left) foot of as follows: (from high to low) was as follows: CG resultant force - CG resultant momentum - Knee angle (R) - Thigh angle (R) - CG horizontal acceleration CG vertical acceleration - Thigh angle (L) - Foot angle (L) - Knee angle (L) Foot angle (R) - CG vertical velocity - CG horizontal velocity. These results prove the second hypothesis. 


\section{Recommendations:}

In the light of this research results and conclusions, the researchers recommend the following:

- Building training program dominant foot and non-dominant foot should be equally trained to improve performance of soccer players.

- Use biomechanical indicators of angles (knee - thigh - foot) - The weight of the body center for the player during Shooting with the Internal Instep as a Basis for Soccer Specific Exercises.

- Use exercises invocation of skills based on the foundations biomechanical in football training programs to improve performance of soccer players.

- Conduct training courses for coaches to study the biomechanics and its practical applications in football skills. 


\section{References:}

1- Abd El-Aziz, Ahmed K. (): Biomechanical variables of the efficiency of short corner kick and movements of attackers in soccer Phd, Faculty of Physical Education-Elmnya University,2009. (in Arabic)

2- Alaa El-Din, G. \& Al-Sabbagh, N. (2007): Metrological bases for evaluation of physical, technical and tactical performance in athletes. Munshaat Al-Maaref Alexandria - Egypt (in Arabic)

3- Alaa El-Din, Gamal M. (1989): Movement systems: its guidance and control. Journal of theories and application - Faculty of Physical Education for Men Alexandria University, Issue (6) (in Arabic)

4- Ali, Ahmed A. M. (): Biomechanical indicators and its relation to effectiveness of performing complex skills on two types of football courts (a comparative study) Master, Faculty of Physical Education-Elmansoura University,2013. (in Arabic)

5- Berekaa, M. \& Al-Sokary, K. (2002): Basics of biomechanics in sport. Munshaat Al-Maaref - Alexandria - Egypt (in Arabic)

6- Hammad, M. \& Kishk, H. (2002): Futsal. Gazirat Al-Ward Press - Mansoura Egypt (in Arabic)

7- Hochmuth Gerard (1999): Biomechanics and methods of studying sports movements. Translation by: Kamal Abdul Hamid \& Soliman Ali Hassan. Markaz Al-Ketab Press - Cairo - Egypt (in Arabic)

8- Hussam El-Din, Talha (1993): Biomechanics: Theoretical and Practical Bases. $1^{\text {st }}$ ED, Dar Al-Fikr Al-Araby - Cairo - Egypt (in Arabic)

9- Mohamady, M. \& Ali, M. (1998): Mental preparation and improving tactical thinking for soccer players. Dar Al-Fikr Al-Araby - Cairo - Egypt (in Arabic)

10- Mokhtar, Hanafy M. (1994): Scientific bases of soccer training. Dar Al-Fikr AlAraby - Cairo - Egypt (in Arabic)

11- Saber, Tamer S. M. (): Contribution percentages of biomechanical variables in performance efficiency of some complex skills for junior soccer players, Master, Faculty of Physical Education-Elmansoura University,2011. (in Arabic) 